\title{
L'ÉVOLUTION D'UN SYNDICAT DU GRAND PARIS : RETOUR SUR VINGT ANS DE TRANSFORMATIONS DU SIPPÉREC
}

Témoignage de Catherine DUMAS

Directrice adjointe du Syndicat intercommunal de la périphérie de Paris pour les énergies et les réseaux de communication (SIPPÉREC) de 2006 à 2016

\author{
recueilli par \\ David GUÉRANGER \\ et François-Mathieu POUPEAU \\ Chercheurs au Laboratoire techniques, territoires et sociétés (LATTS, Université Paris-Est)
}

Quand êtes-vous entrée au SIPPÉREC?

Je suis entrée en 2001. C'est à cette époque que le SIPPÉREC entame sa diversification dans les communications électroniques et que le monde de l'électricité commence sa mutation avec les prémices de l'ouverture du marché de l'électricité à la concurrence ${ }^{1}$. La compétence du syndicat dans les télécom remonte à 1997, au moment de la libéralisation de ce secteur. Le syndicat s'est alors rapidement investi dans la création d'un groupement de commandes pour les collectivités, qui devaient pour la première fois lancer des marchés publics pour satisfaire leurs besoins et faire jouer la concurrence. Il décide également de construire des réseaux câblés et un réseau très haut débit en fibre noire pour lutter contre les inégalités territoriales. Je suis entrée juste à ce moment donc j'ai pu participer à la mise en œuvre de tout cela, lorsque le syndicat s'est fait plus offensif : comment le syndicat s'est préparé à ces ouvertures des marchés, ce qu'il a pu essayer, avec d'autres, d'influencer, l'évolution du rôle des autorités concédantes $^{2}$ après la loi Sapin 1 et l'application du nouveau contrat de concession

\footnotetext{
${ }^{1}$ Il est très difficile, dans le cadre d'un tel article, de faire un point complet sur les compétences et le périmètre géographique du SIPPÉREC. Depuis qu'il a entamé une politique de diversification, dans les années 1990, ce syndicat propose un bouquet de compétences qui dessine une carte de ses adhérents à géométrie variable. À titre d'exemple, le SIPPÉREC compte en 202084 communes adhérentes sur sa compétence historique électricité (qui a motivé sa création, en 1924). En ce qui concerne les réseaux numériques, 90 communes sont concernées, sur 6 départements. Sur les énergies renouvelables, 80 communes adhèrent au syndicat, mais sur un périmètre géographique qui n'a rien à voir avec celui de la compétence électricité. Pour une illustration de cette grande diversité, cf. Emmanuel Bellanger, François-Mathieu Poupeau, Lumières sur la banlieue. Histoire du syndicat intercommunal de la périphérie de Paris pour l'électricité et les réseaux de communication (SIPPÉREC), Paris, Les Éditions de l'Atelier, 2013.

${ }^{2}$ Sur cette compétence des syndicats de communes, voir l'article de F.-M. Poupeau dans ce dossier.
} 
avec EDF signé en 1994, la défense des usagers dans ce nouveau monde, le développement des énergies renouvelables et, plus récemment, les dernières évolutions institutionnelles, que ce soit la loi NOTRe, les intercommunalités ou le Grand Paris.

Les réformes sectorielles et les directives européennes sur l'ouverture des marchés et la décentralisation ont fortement influencé ces évolutions. Par exemple la loi Sapin qui a quand même obligé les autorités concédantes... à remplir leur rôle ! On va dire que, jusqu'aux années 1990, les autorités concédantes - particulièrement dans le domaine de l'énergie mais pas seulement - ne contrôlaient pas grand-chose. C'est à partir du milieu des années 1990 que le SIPPÉREC s'affirme comme un acteur de la régulation du service public de l'électricité et des télécom, développe son expertise, acquiert des compétences internes. Les relations avec EDF se tendent, elles sont l'expression d'une volonté des élus d'avoir un rapport plus équilibré avec le concessionnaire, de vouloir défendre leur territoire et les usagers.

\section{La loi Sapin est pourtant plus connue sur l'eau, et beaucoup moins sur l'énergie...}

Jusqu'au milieu des années 1990, les grands syndicats, que l'on retrouvera alliés trente ans plus tard pour défendre leur particularité et leurs actions en région parisienne, avaient une administration et un siège communs : SEDIF, SIGÉIF, SIPPÉREC, SIFUREP 3 . Le premier à prendre son indépendance, c'est le SIGÉIF, ensuite c'est le SIPPÉREC. En 1994, la signature du nouveau contrat de concession avec EDF ouvre une nouvelle ère pour le syndicat, qui se réapproprie un rôle d'autorité concédante peu exercé précédemment, ce qu'avait dénoncé la Chambre régionale des comptes d'Île-de-France en 1990. À partir de là, le SIPPÉREC structure sa propre administration. C'est l'arrivée d'Étienne Andreux comme directeur général, d'un ingénieur en chef à temps complet et de sept agents et aussi la création d'une cellule technique.

\footnotetext{
${ }^{3}$ Syndicat des eaux d'Île-de-France (SEDIF), Syndicat intercommunal pour le gaz et l'électricité en Île-deFrance (SIGÉIF), Syndicat intercommunal funéraire de la région parisienne (SIFUREP).
} 
Et pourquoi n'était-il pas possible d'exercer son rôle d'autorité concédante en conservant une administration commune?

Parce qu'en particulier, les secteurs de l'énergie nécessitaient de développer une expertise différente de celle de l'eau. Le seul syndicat qui était structuré et qui mettait ses compétences au service des autres, c'était le SEDIF. Ce qui montre bien l'importance du secteur de l'eau. C'était le seul qui avait des moyens financiers, les moyens d'avoir sa propre administration qui était mise à disposition. Mais quand je dis «mise à disposition », c'était le directeur général et un demi-secrétariat qui l'étaient pour gérer le contrat du SIPPÉREC. L'énergie était un secteur en monopole où les autorités concédantes ne contrôlaient pas grand-chose, et où on faisait une totale confiance à « la grande entreprise nationale EDF ».

Avec le nouveau contrat de concession de distribution publique d'électricité, le SIPPÉREC se doit en particulier de gérer au mieux les crédits destinés aux communes adhérentes, résultant des nouveaux accords, et de veiller au respect des engagements pris par EDF. Je pense en particulier à l'enfouissement des réseaux et à la qualité. Le syndicat développe ses compétences, en particulier en matière de contrôle, pour apprécier l'état de la concession et les performances du délégataire. Avec la multiplication des contrats de concession dans le domaine des communications électroniques, puis de géothermie dans une période très récente, et le développement des services aux collectivités, le SIPPÉREC n'a cessé de développer son expertise et donc d'étoffer son équipe : de 10 agents en 2001 à 72 quand je suis partie fin 2016. Ils doivent être plus de 80 aujourd'hui, tout en faisant appel à des expertises extérieures pour certaines missions très pointues.

Pouvez-vous revenir sur la prise de compétence communications électroniques?

S'il est bien un moment important du développement du SIPPÉREC et de sa diversification, c'est l'ouverture à la concurrence du secteur des télécom! En effet, alors que l'ouverture avance lentement dans le secteur de l'énergie, au fil des discussions européennes, le rythme des réformes va beaucoup plus vite dans les télécom, bousculées par nombre d'innovations technologiques. Les frontières s'effacent entre télécom, audiovisuel et informatique.

En 1997, le syndicat prend cette nouvelle compétence et change de nom : le SCBPE [Syndicat des communes de la banlieue parisienne pour l'électricité] devient le SIPPÉREC [Syndicat 
intercommunal de la périphérie de Paris pour l'électricité et les réseaux de communications]. Tout comme dans l'électricité, le syndicat progressera pas à pas, au fur et à mesure que des questions nouvelles se posent pour ses adhérents, que les technologies et la législation évoluent. Le SIPPÉREC commence par lancer des délégations de service public pour créer des réseaux câblés absents d'une partie de son territoire puis, assez rapidement, pour construire des réseaux haut débit fibre (réseaux de collecte et desserte fibre noire puis fibres actives ${ }^{4}$ ), tout en élargissant le nombre des collectivités adhérentes à cette compétence optionnelle. Objectif : éviter un creusement des inégalités territoriales face aux stratégies des opérateurs télécom.

C'est la loi de 1999 qui ouvre timidement la porte aux collectivités locales pour la construction des réseaux haut débit. C'est notamment l'article L. 1511-6 du Code général des collectivités territoriales. Jusque-là, les collectivités se heurtaient à un refus des pouvoirs publics pour protéger l'opérateur historique France Télécom. Face à cela, un certain nombre de collectivités locales en avance sur ces questions se sont regroupées en Comité de liaison pour faire évoluer la réglementation. Il s'agit de la Communauté urbaine du Grand Nancy, du district de Toulouse, du SIPPÉREC et du Conseil général du Tarn. Un premier mémorandum est publié en octobre 1998, qui vise à interpeller l'État et lui demande d'accorder une compétence nouvelle aux collectivités locales : les autoriser à établir des infrastructures de fibre noire sur leur territoire. La loi d'orientation pour l'aménagement et le développement durable du territoire de juin 1999 voit le jour et introduit un nouvel article (L. 1511-6) dans le code général des collectivités territoriales. Je dirais qu'il s'agit de la première pierre, qui a permis au SIPPÉREC de créer son premier réseau très haut débit. La législation a ensuite évolué, toujours sous l'impulsion des collectivités, de leurs associations (le comité de liaison s'est élargi en particulier à l'AVICAM, l'Association des villes câblées), avec la loi sur la confiance dans l'économie numérique votée en avril 2004. Les collectivités peuvent alors établir ou exploiter des réseaux de télécom ouverts au public.

\footnotetext{
${ }^{4}$ La fibre noire est une fibre optique brute installée mais qui n'est pas encore activée.
} 
Sur quelles bases se noue cette initiative commune entre le District de Toulouse, le SIPPÉREC, le Conseil général du Tarn et Nancy?

La Communauté urbaine du Grand Nancy a été la première collectivité locale à avoir eu un projet de construire une infrastructure de fibre noire. Elle s'est alors opposée à France Télécom, qui a tout fait pour l'en empêcher. Il fallait faire évoluer la réflexion dans ce domaine et faire évoluer la loi. Il se trouve qu'à l'époque, ces collectivités avaient les mêmes conseillers techniques, financiers, juridiques, ce qui les a rapprochées.

Cette période marque, semble-t-il, un changement de posture pour le SIPPÉREC, qui devient beaucoup plus offensif, qui va essayer de faire évoluer son environnement législatif et réglementaire, ce qui était moins le cas avant...

Le changement de posture du syndicat se met progressivement en place à partir du milieu des années 1990, avec l'arrivée d'un nouveau Président, de nouveaux élus, et celle d'Étienne Andreux comme Directeur général, la création d'expertises techniques, juridiques, financières autant en interne qu'en externe. Effectivement, c'est l'ouverture du secteur des télécom qui pousse dans un premier temps à cette posture, comme je l'ai expliqué. Puis l'ouverture progressive à la concurrence de l'électricité oblige le syndicat à monter en compétence à partir des années 2000 dans ce domaine également, pour anticiper les évolutions et protéger collectivités et usagers. C'est le moment où je suis arrivée au syndicat. Les élus ont voulu comprendre en allant voir ce qui se passait dans les pays étrangers, en particulier en Angleterre, plus avancée sur l'ouverture du marché de l'électricité. Ces compétences de très haut niveau qu'on avait mobilisées dans le domaine des télécom, nous les avons trouvées aussi auprès de consultants dans le domaine de l'électricité. La direction du syndicat opérait un système de veille. Mais on était aussi alimenté par un travail permanent avec des acteurs extérieurs.

De ce point de vue, comment a évolué le rôle joué par le service juridique?

La montée en compétence du service juridique en interne a été très importante dans les dix dernières années. Elle est due en particulier à l'augmentation du nombre de contrats de concession à gérer. On est parti de la gestion d'un seul contrat de concession en électricité en 
1994, à vingt-cinq contrats aujourd'hui dans les télécom et la géothermie. Elle est aussi liée au fait que le syndicat a développé des compétences différentes, a créé des groupements d'achats dans les télécom, dans l'électricité, puis dans les énergies renouvelables et les SIG [systèmes d'information géographique]. Toutes ces compétences et tous ces services développés pour faciliter la vie des collectivités adhérentes ont nécessité d'étoffer l'équipe et l'expertise du syndicat, y compris dans les marchés publics, dont la réglementation n'a elle aussi cessé d'évoluer.

C'est lié aussi aux contentieux qui concernent notamment EDF et les nombreux opérateurs?

Ce n'est pas notre service juridique qui va plaider devant le tribunal administratif ni devant le Conseil d'État. C'est un cabinet juridique extérieur voire plusieurs selon les juridictions qui travaillent ces dossiers, en lien étroit avec les élus, la direction et le service juridique du syndicat.

D'autres lois que la loi Sapin ont-elles eu un effet analogue sur l'organisation du SIPPÉREC, sur le développement de compétences administratives singulières?

Plus les lois concernant nos secteurs d'activités, tant énergie que télécom, ont évolué entre les années 1995 et 2010, plus les collectivités ont été autorisées à investir de nouveaux champs d'action, plus le SIPPÉREC a développé des services aux collectivités et a agrandi son territoire. C'est ainsi que le syndicat s'est doté de compétences internes nouvelles. Quand je suis entrée au SIPPÉREC, il y avait dans l'équipe technique trois ingénieurs électricité et un ingénieur télécom, point final. Avant que je ne parte, nous avons embauché, par exemple, cinq ingénieurs en énergies renouvelables. On a créé un service d'enfouissement des réseaux au moment où l'on a repris la maîtrise d'ouvrage dans ce domaine, avec cinq personnes affectées. En matière de télécom, c'est pareil, nous avons démarré avec un ingénieur. Aujourd'hui, il doit y en avoir cinq pour gérer une dizaine des délégations de service public. L'augmentation du personnel et des compétences internes suit l'évolution des services aux collectivités adhérentes, plus diversifiés. 
Cette augmentation tendancielle du nombre de postes a-t-elle rencontré des résistances, des problèmes ou des difficultés, budgétaires par exemple?

Nous avons toujours essayé de développer des compétences en faisant en sorte qu'elles s'autofinancent. Le SIPPÉREC vit uniquement avec ses redevances de concessions, avec les coûts d'adhésion aux groupements de commandes ou avec un pourcentage sur les services exercés, comme par exemple le contrôle de la taxe locale d'électricité ${ }^{5}$. Le syndicat ne dépend pas de la fiscalité locale. Ceci a toujours obligé le syndicat à faire le bilan financier des services apportés aux collectivités. Par exemple, on a pris à un moment donné, à la demande de certaines de nos collectivités qui y voyaient une possibilité de meilleure gestion et d'économies, une compétence éclairage public et signalisation lumineuse tricolore. Au moment où l'on a proposé cette compétence se sont créées les communautés d'agglomération, et ces questions ont été traitées à ce niveau. On est certainement arrivé un peu tard avec nos propositions, dans un secteur très concurrentiel et qui nécessite beaucoup de proximité. Faute de rentabilité, les élus ont décidé de laisser de côté cette compétence. Pour le reste, l'augmentation de l'effectif a conduit à des problèmes de locaux et un déménagement deux mois avant mon départ, et à une évolution dans le management bien évidemment.

Avez-vous rencontré des problèmes de recrutement sur ces compétences nouvelles?

Il n'est pas toujours évident d'attirer des compétences pointues dans le secteur des collectivités, qui n'offre pas les rémunérations du privé ni les mêmes perspectives d'évolution de carrière. Il faut «vendre les atouts du secteur public » à des candidats qui souvent en ignorent tout. Ce sont de nouveaux métiers pour les collectivités : par exemple recruter des ingénieurs spécialisés en solaire, géothermie, hydrogène, capables de contrôler des délégations de service public pour les télécom ou de proposer des services dans le cadre de la ville connectée. Tout cela, ce sont des métiers qui n'existent pas ou très rarement en collectivité. C'était plutôt ça notre problématique : comment arriver à attirer dans un service public des compétences qui n'existent que dans le privé ? Avec les limites du statut et de la rétribution du secteur public. Nous avons réussi à embaucher beaucoup de jeunes, surtout dans le secteur des énergies renouvelables, qui avaient deux ou trois ans d'expérience et qui, 
en venant chez nous, avaient une fibre un peu service public. Certains ont passé avec succès les concours de la fonction publique et une grosse partie d'entre eux sont devenus ingénieurs de la territoriale. C'est aussi notre fierté. Nous avons toujours mis des moyens dans la formation du personnel pour que les agents passent et obtiennent les concours aussi bien dans la filière technique qu'administrative.

Quelles sont rétrospectivement les grandes réformes qui ont eu les répercussions les plus importantes sur le SIPPÉREC?

En dehors des réformes sectorielles, je dirais que ce sont toutes les réformes liées à la décentralisation et l'intercommunalité. Les premières qui influent, ce sont celles qui obligent à la création de communautés d'agglomération aussi en région parisienne, avant qu'on arrive à l'époque actuelle avec le Grand Paris et la création de la MGP [Métropole du Grand Paris]. À partir du moment où il y a eu la création des communautés d'agglomération, la réflexion du syndicat et de ses élus a été, bien sûr, l'élargissement de ses compétences sectorielles, mais aussi une réflexion sur l'extension de son territoire. C'est l'adhésion des premières communautés d'agglomération au SIPPÉREC, la première étant celle de Val de France à la compétence énergies renouvelables. Puis celle des départements : l'Essonne et le Val de Marne puis, fin 2016, celle du Conseil Régional Île-de-France. L'élargissement du territoire s'est d'abord fait sur les compétences optionnelles (télécom et énergies renouvelables), sur les groupements de commandes (télécom, électricité, SIG). Puis les élus ont décidé aussi de proposer la compétence électricité à d'autres collectivités qui géraient encore seules des contrats de concession et ne disposaient pas des compétences internes nécessaires.

${ }^{5}$ Celle-ci est prélevée sur les factures d'électricité. 
Comment s'est déroulée l'adhésion de la communauté de Val de France, dont vous avez parlé ? Comment on passe d'un syndicat de communes à un syndicat qui ne va plus seulement être de communes?

À chaque fois, cela a nécessité de modifier les statuts. Avec l'adhésion de la Communauté d'agglomération de Val de France, le syndicat de communes est devenu un syndicat mixte fermé, puis avec l'adhésion du département et de la Région, un syndicat mixte ouvert ${ }^{6}$. C'est un processus très long qui nécessite la délibération de chaque adhérent et un arrêté interpréfectoral. L'objectif est toujours de répondre aux besoins nouveaux des collectivités, souvent démunies devant l'évolution des technologies et de la réglementation, pour réussir la transition numérique et énergétique. Il s'agit de leur offrir des services mutualisés, d'optimiser les coûts et de soutenir l'investissement. Il est évident que certains sujets comme les réseaux télécom ne se réfléchissent pas à l'échelle d'une commune. La réflexion et la construction se sont toujours faites à l'échelle intercommunale. Les liens développés avec toutes les collectivités des groupements de commandes ont aussi poussé les élus à cette réflexion sur le territoire. Quand on se retrouve avec 300 collectivités adhérant au groupement de commandes télécom, le syndicat a déjà largement dépassé son territoire de compétence initiale.

D'où vient cette nécessité impérieuse d'élargir, de conquérir de nouveaux territoires, de trouver de nouveaux partenaires?

Les élus à la tête du syndicat sont eux-mêmes élus au sein de communautés d'agglomération, de départements, de la région et sont donc confrontés à ces évolutions territoriales et institutionnelles. Quand l'élargissement a été décidé dès la mise en place de la compétence télécom au milieu des années 90, on ne parlait pas encore de la Métropole du Grand Paris [MGP] ! Puis est venue la création des communautés d'agglomération, de la MGP.

Le SIPPÉREC est un acteur incontournable dans le domaine de l'électricité, la plus importante concession en France. Puis il l'est devenu dans le développement des énergies renouvelables, comme premier acteur régional, et, bien sûr, il tient un rôle majeur dans l'aménagement

\footnotetext{
${ }^{6}$ Voir le glossaire en fin d'introduction de ce dossier pour l'explication de ces formes d'intercommunalités.
} 
numérique de l'Île-de-France. Pour les élus, le SIPPÉREC remplit un rôle unique, de proximité, indispensable pour le développement du territoire, la protection des usagers, l'investissement et, à ce titre, ils souhaitent qu'il continue à se développer et ne soit pas englobé dans une super technostructure.

La deuxième raison est que, depuis plusieurs années, les élus ont compris que face aux groupes puissants qu'ils avaient en face d'eux, EDF, France Télécom devenu Orange, le groupe Louis Dreyfus, Numericable, SFR etc., face à tous ces acteurs-là, il fallait se regrouper pour peser dans les négociations. Plus le territoire était important, plus le syndicat était un acteur reconnu en Île-de-France, plus il allait pouvoir défendre l'intérêt des collectivités adhérentes.

Il n'y avait pas de contradiction finalement, entre vos stratégies sectorielles et votre stratégie institutionnelle...

Non, elles sont intimement liées.

Avez-vous eu en tête l'idée qu'à terme, les communes puissent être remplacées par des communautés de communes et d'agglomération en tant qu'adhérentes?

Tout dépend des compétences exercées par chaque strate de collectivités au fil des évolutions législatives. La compétence de l'électricité est restée à l'échelon communal, en tout cas, en région parisienne. Les communautés d'agglomération sont généralement compétentes en matière d'énergies renouvelables et de maîtrise de l'énergie, les départements ou communautés d'agglomérations en matière de télécom. Le SIPPÉREC s'est adapté et a accueilli ces différentes strates de collectivités au fur et à mesure.

Avez-vous aussi développé une action de veille voire de lobbying?

Comme je l'ai dit, le syndicat l'a toujours eue dans les secteurs dont il s'occupe. Concernant l'évolution de la Métropole du Grand Paris, il est clair qu'il y a eu un lobbying des syndicats de la région parisienne. Il s'est créé dans les dernières années, juste avant que je ne parte, une 
conférence intersyndicale entre les syndicats des grands services publics urbains de la région Parisienne : SIAAP, SIGÉIF, SIPPÉREC, SYCTOM, EPTB Seine Grands Lacs, SIFUREP7 Cette conférence intersyndicale, sa genèse, c'est de défendre la spécificité de ces grands syndicats alors que se discutaient la loi NOTRe et la loi MAPTAM, qui oubliaient totalement qu'en région parisienne, la construction des services publics locaux était différente. Donc, effectivement, il y a eu une bataille des élus, des députés, des sénateurs, dont un certain nombre étaient élus dans ces syndicats, pour que les syndicats gardent leur spécificité. Une bataille contre les pouvoirs publics qui s'est terminée de nuit à l'Assemblée !

Est-ce qu'il y avait des différences ou des points sur lesquels vous n'étiez pas immédiatement en accord entre syndicats?

Non. Après, chaque secteur d'activités a des problématiques différentes. L'EPTB avait des problématiques différentes de celles du SYCTOM, etc. Mais, pour se battre ensemble pour permettre à ces syndicats de continuer à exister et à développer leurs compétences, à défendre cette spécificité en région parisienne, on s'est tous retrouvé très vite. Il n'y a pas eu d'achoppement, alors même que les couleurs politiques des présidents des syndicats, quand cela a commencé, étaient différentes. Le bénéfice de tout cela, c'est que les syndicats se sont enfin rencontrés, ils se sont enfin parlé. Et, depuis, des projets communs se développent, par exemple entre le SIPPÉREC et le SYCTOM. Nous avons réappris à nous connaître, à renouer ces alliances qui existaient avant les années 1990.

Pourrait-on revenir sur les scénarios envisagés au moment de la création de la Métropole du Grand Paris, tels qu'ils avaient été mis sur la table?

L'État a voulu à un moment donné que la Métropole du Grand Paris, comme les communautés urbaines, reprenne les compétences eau, énergie, assainissement. Sans prendre en compte le fait qu'en région parisienne, il y avait une organisation, le SIPPÉREC, qui s'était mise en place depuis le début du vingtième siècle, s'était structurée et avait des compétences

\footnotetext{
7 Syndicat interdépartemental pour l'assainissement de l'agglomération parisienne (SIAAP), Syndicat mixte central de traitement des ordures ménagères (SYCTOM), Établissement public territorial de bassin (EPTB).
} 
propres. Cela n'existait pas de cette façon ailleurs. J'ai participé à ces réunions, je suis allée avec les élus dans les ministères rencontrer les cabinets pour leur expliquer qui on était. Ils ont découvert qu'on existait. Le SIPPÉREC par exemple est connu du microcosme de l'énergie et des télécom, des ministères concernés à certaines époques... Mais, comme l'administration change régulièrement, au moment de la loi MAPTAM, personne ne connaissait ces syndicats. Il a fallu publier un mémorandum pour expliquer leur légitimité, leur histoire, leurs actions.

Cela veut dire concrètement que le ministère de l'Intérieur, et en particulier sa Direction générale des collectivités locales, vous ne les côtoyiez pas avant?

Au moment des textes de loi sur les télécom, bien sûr que nous avons été dans les ministères. Sur l'énergie, quand le syndicat a attaqué le TURPE [tarif d'utilisation des réseaux publics d'électricité] par exemple, les ministres de l'énergie ou de l'Industrie nous connaissaient, parce qu'ils recevaient régulièrement des lettres du SIPPÉREC, mais sur des questions qui étaient très sectorielles. Je me souviens m'être rendue au ministère de l'Énergie quand le syndicat s'est battu sur la précarité énergétique, mais, là aussi, c'était sur des questions très ponctuelles, et les cabinets ministériels, ça change ! Et ceux qui s'occupaient de ces questions ne sont pas ceux qui travaillaient sur les textes de lois NOTRe ou MAPTAM !

\section{Côtoyiez-vous les préfets?}

Oui, parce que, de toute façon, le syndicat est une collectivité territoriale dont les actes sont contrôlés par la préfecture. Donc certaines questions nécessitent le dialogue avec le préfet voire les préfets des départements sur lesquels le syndicat agit.

Au moment de la MGP, avez-vous essayé de jouer de vos relations avec le préfet pour essayer de faire entendre votre voix?

Tout à fait. À la fois le Président du syndicat en propre, et à la fois grâce à cette fameuse intersyndicale. 
Pouvez-vous nous en dire plus sur le travail que vous avez mené auprès des élus?

C'est un travail de proximité avec le président, les vice-présidents, les délégués pour expliquer des problématiques qu'ils ne rencontrent pas dans leurs collectivités puisqu'ils nous ont confié ces compétences. Ce sont des sujets complexes, qui nécessitent beaucoup d'investissement de leur part. Quand je suis arrivée au SIPPÉREC, seul le président était encore maire et président d'une communauté d'agglomération, Plaine Commune. Les maires avaient déserté le conseil syndical au fur et à mesure que l'électrification de la région parisienne s'était achevée. L'électricité était un monopole. Une fois, la compétence déléguée au syndicat, il n'y avait plus grand chose à négocier. Les maires s'en sont désintéressés. On avait comme représentants des collectivités quelques adjoints, des conseillers municipaux. Comme c'étaient des questions très techniques, cela n'intéressait pas grand monde. Ces dernières années, avec les nouvelles compétences et la montée en puissance des questions numériques, d'aménagement du territoire, de précarité énergétique et de développement durable, les maires ont recommencé à s'intéresser au syndicat et à participer aux instances. Aujourd'hui, dans le bureau, il y a cinq maires. Cela ne s'était plus vu depuis longtemps. C'est important pour débattre de la stratégie du syndicat et de son rôle dans le nouveau paysage institutionnel.

Vous avez mobilisé ces élus des communes au moment de la création de la Métropole du Grand Paris?

Ils se sont un peu mobilisés tout seuls parce qu'ils se sentaient concernés en tant que maires et qu'ils étaient tous déjà dans les instances de réflexion sur la création de la Métropole. Et, pour un certain nombre, ils étaient des acteurs de premier plan dans ces réflexions. Je pense par exemple au président actuel Jacques JP Martin, à l'ancien maire d'Arcueil, Daniel Breuiller, vice-président du SIPPÉREC. Je pense à quelques élus comme cela, un peu emblématiques des réflexions sur la Métropole.

Comment votre travail de mobilisation des maires se déroulait-il concrètement?

Ce sont des matinées d'informations, des colloques que le syndicat organisait régulièrement. C'est aussi du travail de terrain, nombre de rendez-vous avec les maires, les principaux 
adjoints, pour leur présenter nos nouvelles compétences, nos nouveaux services, les intéresser à nos questions, répondre surtout à leurs préoccupations pour anticiper leurs besoins. Quand on travaille par exemple sur la géothermie, on le fait à l'échelle d'une, deux, voire trois ou quatre collectivités, et c'est du travail permanent avec les maires, en particulier lors de la construction des centrales géothermiques, qui nécessite beaucoup de dialogue de proximité avec les riverains et les habitants.

Aviez-vous une stratégie de ciblage des élus qui vous semblaient clés, sur lesquels il fallait un peu plus travailler pour leur dire ce vous faisiez?

Cela dépend des époques et des sujets. C'est vrai que, par rapport au lobby parlementaire et aux différentes époques où on a eu besoin d'influer sur la législation, on a travaillé avec des maires et parlementaires présents sur notre territoire voire au-delà. Quand le syndicat a décidé d'agrandir son territoire, bien sûr il y a eu un travail pour essayer d'aller convaincre telle ville ou tel maire d'adhérer.

Pour ce travail, quelles sont les personnes impliquées?

Il y a le président et les élus du bureau, le directeur général, moi-même.

Est-ce que les associations d'élus ou les associations d'institutions locales, à un moment, ont été des espaces aussi investis par le SIPPÉREC?

Bien sûr. Quand on parle des partenaires à différents moments, les associations d'élus en font partie. On a beaucoup travaillé au moment de l'évolution des lois sur les télécom avec l'Association des maires des grandes villes de France. On a également beaucoup travaillé avec l'Association des Maires d'Île-de-France, l'AMIF, avec l'AdCF [Assemblée des communautés de France]. Nous avons aussi travaillé en matière d'énergie et de télécom avec la FNCCR [Fédération nationale des collectivités concédantes et régies], avec l'AVICA [Association des villes câblées] dont je parlais tout à l'heure, avec l'Association de géothermie d'Île-de-France, l'AGEMO. Bien sûr, nous avions un champ d'acteurs institutionnels avec lequel le syndicat a 
collaboré et avec qui nous avons été plus ou moins d'accord, suivant les périodes. Dans les dernières années, le syndicat a aussi beaucoup travaillé avec les associations de consommateurs, et en particulier UFC Que Choisir. Je crois important à ce stade d'expliquer que si le SIPPÉREC a mené certains contentieux, en particulier face à EDF et ERDF [devenu depuis ENEDIS] dans les quinze dernières années, c'est pour défendre l'intérêt des collectivités et des usagers. Je pense que c'est important de montrer que des institutions comme le syndicat ont des compétences, se mobilisent, se donnent les moyens d'aller vaincre un peu l'opacité des grands groupes que nous avons en face. Tout cela, on ne le fait pas pour nos beaux yeux, on le fait pour le service public, pour défendre l'intérêt des territoires et des citoyens. Dans le débat actuel, je pense que c'est important de rappeler cette dimension-là de l'action des syndicats.

Quelle était la nature des arguments que vous opposiez à l'intégration de vos compétences au sein de la Métropole du Grand Paris?

À l'échelle de la métropole, regrouper demain tous ces gros syndicats, cela va rapidement être « une usine à gaz » qui ne pourra plus se mettre en mouvement. Quelque part, le succès du SIPPÉREC, qu’Étienne Andreux a qualifié pendant longtemps de «start-up territoriale», c'est une agilité dans la réflexion, dans la mise en œuvre, dans la mise en pratique. Avec une ligne hiérarchique courte, des décisions rapides, une proximité avec ses adhérents. Tout cela, dans une structure englobée à la métropole, nous risquons de le perdre.

Le travail en commun peut se faire sous d'autres formes et il se fait déjà. Cela fait longtemps que le SIPPÉREC a développé avec la Ville de Paris des relations de travail et d'échange sur le contrat de concession : avant, pendant ou après le renouvellement des contrats. On peut toujours discuter de ce que les uns et les autres ont obtenu ou lâché mais les échanges d'expériences, cela fait maintenant presque une dizaine d'années qu'ils se sont mis en place.

Ces réformes institutionnelles sont aussi des opportunités. On a parlé de la conférence intersyndicale mais, dans le même temps, tous les syndicats d'énergie d'Île-de-France ont créé un Pôle Énergies en 2016, juste au moment où je suis partie. Il regroupe le SIPPÉREC, le SIGÉIF, le syndicat de Seine-et-Marne, des Yvelines et du Val-d'Oise. Parce que la menace, à un moment donné, a été effectivement de n'avoir qu'un seul syndicat d'énergie à l'échelle de la région parisienne. L'idée, c'était déjà de se rencontrer, d'essayer de partager nos 
expériences. Et puis les plus aguerris vont essayer d'aider ceux qui ont moins d'expérience. Alors, après, regrouper demain à l'échelle de l'Île-de-France, au moins à l'échelle de la première couronne, les trois contrats de concession de Paris, SIPPÉREC, SIGÉIF, c'est EDF et ENEDIS qui ne seront pas forcément d'accord!

Comparé à l'expérience que vous avez évoquée avec la communauté d'agglomération de Val de France, il semble y avoir dans ce cas une stratégie un peu différente?

Disons que l'on n'est pas à la même époque. Quand le syndicat intègre Val de France, on est bien avant la création de la Métropole, etc. Les syndicats ne sont pas encore menacés. La réflexion est : « si on veut développer des compétences, si on veut mutualiser à l'échelle d'un territoire de plus en plus de services, il faut agrandir notre territoire et tenir compte des compétences de nouvelles institutions ». Dans la période plus récente, on est plutôt dans « ils nous attaquent, montrons qui nous sommes, ce que nous avons réalisé et développons chaque jour », avec la conférence intersyndicale et la création du Pôle Énergies Île-de-France. Et puis les choses bougent, un petit syndicat d'électricité constitué d'une commune de l'Essonne et de 4 communes du Val de Marne, SUD-ELEG, vient d'adhérer au syndicat. C'était une structure sans personnel, sans moyens de contrôle. Cela faisait au moins dix ans que le syndicat l'avait approchée. Il y a quand même une évolution des mentalités, y compris politiquement.

Comment voyiez-vous le rôle du SIPPÉREC par rapport au Conseil régional d'Île-deFrance?

Les liens avec le Conseil régional ont été souvent tendus en matière de télécom, l'action du syndicat n'étant pas toujours comprise par les équipes en place. Le lobby des opérateurs y était puissant. Par contre, ces dernières années, en matière d'énergies renouvelables, la Région a décidé de s'appuyer sur le SIPPÉREC et y a adhéré pour une meilleure gestion des installations photovoltaïques existantes et pour en développer de nouvelles, en particulier sur les lycées. La Région a fait des annonces sur sa stratégie régionale en matière d'énergie, en juillet 2018. 15 partenaires se sont engagés autour de la Région pour porter la part des énergies renouvelables de $10 \%$ à $40 \%$ d'ici 2050 , doubler la quantité produite en Île-de- 
France d'énergies renouvelables et réduire de $20 \%$ la consommation énergétique. Le SIPPÉREC fait partie de ces 15 acteurs.

Et qu'est-ce qui explique, sur cet exemple des énergies renouvelables, que vous ayez voulu créer une structure nouvelle (une Société d'économie mixte) plutôt que d'intégrer au SIPPÉREC de nouveaux services?

La réflexion est venue lorsque nous avons commencé à acquérir suffisamment d'expérience en matière d'énergies renouvelables. Nous avons commencé à construire et à exploiter des centrales solaires sur les bâtiments. À un moment donné, là aussi, l'objectif a été de devenir un acteur de poids face à EDF. Si on veut renégocier le contrat de concession, si on veut pouvoir discuter, pas à armes égales mais avec certaines armes face à EDF, il faut qu'on soit producteur d'énergie. D'où la réflexion sur les énergies renouvelables. La SEM [Société d'économie mixte], c'était pour avoir un outil facile qui puisse correspondre à certaines problématiques, qui puisse faire ce qu'un syndicat ne peut pas faire réglementairement et aussi lui permettre d'être producteur d'énergie en dehors du territoire d'Île-de-France, qui est quand même assez contraint en matière de choix de production d'énergies renouvelables, et de pouvoir aussi répondre à des projets citoyens. Là, pour une fois, nous n'avons pas été les premiers. Sur la SEM, on avait des syndicats d'énergie «cousins » qui l'ont fait bien avant nous et on a été voir ce qu'ils faisaient. Nous nous sommes inspirés de leurs expériences.

Nous avons aussi créé une Société publique locale spécifique à Grigny/Viry. Là, c'est un souhait des élus, avec lesquels nous avons travaillé sur le projet de géothermie pendant de longues années, lié à toutes les opérations de rénovation urbaine du quartier de la Grande Borne, donc très sensible politiquement et socialement. Les élus ont vraiment souhaité une SPL pour pouvoir sortir un coût d'énergie le plus bas possible. Et nous avons prouvé que c'était possible puisqu'aujourd'hui cela fonctionne.

\section{Et sur les réseaux dits intelligents (smart grids)?}

Les réseaux intelligents, c'est une réflexion que nous avons engagée deux ou trois ans avant que je parte, et qui se poursuit. Il y a à la fois des choses faites à travers les groupements de commandes télécom et énergie, en particulier pour permettre aux collectivités de s'armer, de 
se doter de réseaux intelligents. Et accompagner de façon pragmatique les collectivités en assurant une veille active, en proposant des rencontres pour partager des expériences.

Avec cette multitude de compétences et d'interlocuteurs, on a un peu le sentiment que depuis les réformes des années 2000-2010, votre environnement est devenu beaucoup plus complexe...

C'est clair que le paysage s'est complexifié au fil des années. C'est évident d'abord dans nos différents secteurs d'intervention. Les lois ont évolué, les acteurs se sont multipliés pour quelquefois se regrouper ensuite ou disparaître. Le syndicat avait le choix de prendre telle ou telle compétence ou service. Le paysage institutionnel, lui, s'impose au syndicat. Je ne sais pas ce que va devenir la MGP mais si nous n'avions pas tous fait le lobbying nécessaire pour que la MGP n'ait pas les compétences réseaux, la discussion que nous avons aujourd'hui ne serait plus la même.

Pour finir, qu'auriez-vous à répondre à celles et ceux qui disent qu'aujourd'hui, les syndicats de communes sont obsolètes face aux autres formes d'intercommunalité (communautés de communes, d'agglomération, urbaines, métropoles)?

Il ne s'agit pas d'opposer une forme d'intercommunalité à une autre. Les syndicats intercommunaux ont été la première forme d'intercommunalité. Dans nombre de domaines, elle a fait ses preuves. Certains, il est vrai, sont ou étaient des coquilles vides mais d'autres ont montré leurs compétences, ont su développer des services publics indispensables aux citoyens. Il ne faut pas généraliser mais plutôt se poser la question : " pour tel service, quel est le territoire pertinent et quels moyens on se donne pour exercer la compétence ? » Au moment où nos concitoyens demandent des services publics de proximité, le débat est ouvert ! 